\title{
Intensidad de dosis relativa de la quimioterapia neoadyuvante en pacientes ancianas con cáncer de mama
}

\author{
Relative dose intensity of the neoadjuvant chemotherapy in elderly \\ patients with breast cancer
}

- Tomás Sánchez Villegas', Jesús Sánchez²

'Internista, oncólogo clínico. Clínica Vida (Medellín, Colombia).

2 Internista, oncólogo clínico. Magíster en epidemiología clínica. Instituto Nacional de Cancerología (Bogotá, D.C., Colombia).

\section{Resumen}

Antecedentes: el cáncer de mama es de los tumores malignos más frecuentes en el mundo. En la población mayor de 65 años se aglomeran la mayoría de las muertes y de supervivientes por esta enfermedad.

Objetivos: describir la intensidad de dosis de la quimioterapia neoadyuvante en ancianas, los factores que la modifican o las consecuencias de alcanzar una intensidad de dosis subóptima.

Materiales y métodos: se realizó un estudio observacional descriptivo retrospectivo. El objetivo principal fue describir la intensidad de dosis relativa de la quimioterapia neoadyuvante en pacientes mayores de 65 años con cáncer mamario del Instituto Nacional de Cancerología que recibieron quimioterapia neoadyuvante entre agosto de 2013 y septiembre de 2015 mediante la revisión de historias clínicas. Se estimó la intensidad de dosis sumatoria de la quimioterapia con base en la fórmula de Hryniuk. Se hizo un análisis exploratory bivariado para evaluar factores asociados a la intensidad de dosis.

Resultados: entre agosto de 2013 y septiembre de 2015 se presentaron 72 pacientes que cumplían los criterios de inclusión y exclusión. El 44,4\% se presentaron en estadio IIIB. El esquema de quimioterapia más utilizado fue el AC-T con un 45,8\%, un $38,8 \%$ de las pacientes tuvieron una intensidad de dosis $\geq 85 \%$. La tasa de respuesta patológica completa global fue de un 16,39\%. La respuesta patológica completa fue más alta en pacientes con sobreexpresión HER2 23,5\%, en triple negativo fue del $23 \%$ y luminal del $11,36 \%$.

Conclusión: en este estudio se mostró que las ancianas reciben con frecuencia una intensidad de dosis de la quimioterapia neoadyuvante subóptima. Adicionalmente, se observó una tendencia a que las ancianas con intensidad de dosis baja mostraran disminución en la respuesta patológica completa, en especial los tumores más sensibles a la quimioterapia citotóxica.

Palabras clave: neoplasias de la mama, quimioterapia, terapia neoadyuvante, anciano.

\section{Summary}

Background: Breast cancer is the most frequent malignant tumor in the world. People older than 65 years represent a group, which gathers most of the deaths and survivors of this disease. It has been published previously that elderly people often receive a suboptimal cancer treatment.

Objetive: Describe dose intensity of the neoadjuvant chemotherapy in the elderly people, the factors that modify this parameter or the consequences of having suboptimal dose intensity.

Methods: This is an observational study descriptive retrospective of breast cancer patients older than 65 years old that received neoadjuvant chemotherapy in the Instituto Nacional de Cancerología between 2013 and 2015. We did a medical record review and estimated the summation dose intensity with the previously published Hryniuk's formula. We also did an exploratory bivariate analysis to find conditions that could modify the dose intensity value.

Results: Between august of 2013 and september of 2015, 72 patients were included. $44.4 \%$ of the patients presented in stage IIIB. The most frequent used chemotherapy scheme was AC-T in $45.8 \%$ of cases. El $38.8 \%$ reached the threshold of $85 \%$ or more. The global pathological complete response was of $16.39 \%$. The biggest pathological response was in patient with overexpression of HER2 the response was $23.5 \%$, and the triple negative subgroup was $23 \%$ and luminal was $11.36 \%$. Conclusion: Our study supports the fact that elderly people often receive a suboptimal antineoplastic treatment. We couldn't demonstrate a demographic or clinic factor that could influence the dose intensity of this population, nevertheless we found a trend towards a lower complete pathological response in the lowest dose intensities especially in the most chemo sensitive tumors.

Keywords: Breast neoplasms, chemotherapy, neoadjuvant therapy, elderly.

\section{Recibido: 26 de febrero de 2019; aceptado: 20 de agosto de 2019}




\section{Introducción}

El cáncer de mama es la neoplasia maligna más frecuente a nivel mundial, y representa la principal causa de muerte por enfermedades neoplásicas en los países menos desarrollados. Para 2018 se presentaron 2.088.846 de casos nuevos y mortalidad de 626.6791,2. Según el último reporte mundial de envejecimiento de la OMS, el porcentaje aproximado de personas mayores de 60 años está entre el $20 \%$ y $24 \%$ para Colombia y los países aledaños; sin embargo, según las proyecciones del cambio de la pirámide poblacional, este número podría superar el 30\% en 2050, cifra únicamente alcanzada por la República de China en este momento ${ }^{3,4}$.

En los Estados Unidos se estima que el $70 \%$ de todos los diagnósticos de cáncer se harán en personas de 65 años o más; además, en este grupo se acumulan más de la mitad de las muertes por esta causa, ${ }^{5,6}$. Se ha publicado previamente cómo las mujeres mayores de 85 años tienen 3 veces más incidencia y 13 veces más mortalidad comparadas con las mujeres entre 40 y 44 años? $^{7}$.

Existen diferencias en el tratamiento de las pacientes ancianas con cáncer de mama frente a las más jóvenes. Las comorbilidades, la percepción de una menor tolerancia al tratamiento, menor acceso a cuidados o la preferencia del médico y/o la paciente y la competición de mortalidad son variables que afectan las decisiones terapéuticas en el paciente anciano ${ }^{6,8}$. Múltiples estudios han demostrado que las pacientes ancianas tienen menos probabilidad de recibir un tratamiento oncológico con intención 'definitiva' o potencialmente curativa versus sus contrapartes más jóvenes ${ }^{9-11}$. Más preocupante aún, existen reportes como los de la doctora Owusu donde los ancianos tienen un $21 \%$ menos probabilidad de ser tratados de acuerdo con las guías, demostrando al mismo tiempo un aumento de 1,8 veces el riesgo de muerte derivado de las variaciones en el tratamiento, luego del análisis multivariado ${ }^{12}$. Así mismo, Allemani en su análisis Eurocare-3 con un diseño similar demostró una baja adherencia al manejo estándar, en este caso un menor uso de la cirugía conservadora de seno y radioterapia adyuvante ${ }^{13}$.

La quimioterapia neoadyuvante ha demostrado impactar en la disminución de la carga tumoral, mejorar el resultado quirúrgico y las complicaciones posoperatorias manteniendo el beneficio en supervivencia que brinda la quimioterapia posterior a la cirugía ${ }^{14,17}$. Para maximizar los beneficios del tratamiento con quimioterapia es necesario cumplir con la dosis correcta en el tiempo correcto, concepto que se ha medido en el cálculo de la intensidad de dosis sumatoria ${ }^{18}$. Desde 1981 Banadonna y Valagussa expusieron la existencia de una relación directa entre la intensidad de dosis de la quimioterapia y la respuesta clínica, encontrando diferencias entre aquellos que tenían lo que denominaron un nivel de dosis (razón entre lo que deberían haber recibido y lo que recibieron), mayor al $85 \%$ versus menor al 65\% ${ }^{19}$. En 1986 Hrynuik publicó su ecuación de la intensidad de dosis, usada hoy en día, lo que permitió hacer un cálculo estándar de la dosis y tiempo ${ }^{20}$.

Más adelante, se publicaron innumerables trabajos que corroboraron las hipótesis de Banadonna y Valagussa, a tal punto de que para hoy constituye una medida estándar para evaluar la calidad de la administración del tratamiento ${ }^{21,22}$.

No existen estudios que evalúen la calidad del tratamiento que se brinda a los ancianos, aun en el contexto de la quimioterapia neoadyuvante. Este estudio pretende hacer una descripción de la intensidad de dosis de la quimioterapia neoadyuvante para cáncer de mama en ancianas; así mismo, describir las variables que puedan estar en relación con este parámetro.

\section{Materiales y métodos}

Se realizó un estudio observacional descriptivo retrospectivo. Se incluyeron pacientes de 65 años o más, con diagnóstico histopatológico de cáncer de mama que recibieron tratamiento neoadyuvante con quimioterapia en la unidad funcional de cáncer de seno del Instituto Nacional de Cancerología entre agosto de 2013 y septiembre de 2015 . Se excluyeron los pacientes con enfermedad metastásica. La información se recogió en un instrumento diseñado para el estudio, obteniendo los datos de la historia clínica sistematizada mediante la técnica de doble digitación para verificar la confiabilidad de la información. Se realizó un análisis estadístico de acuerdo con la naturaleza de la variable. Para variables cualitativas, se midieron frecuencias absolutas y relativas; para variables cuantitativas, se estimaron medidas de tendencia central y de dispersión. La intensidad de la dosis se estimó utilizando la fórmula de Hryniuk ${ }^{11}$.

Además del análisis descriptivo, se realizó un análisis exploratorio bivariado mediante prueba exacta de Fisher para evaluar posibles factores asociados con una 
intensidad de dosis inferior al 85\%.

El análisis estadístico de la información se realizó en el software Stata 11.0. licenciado para el Instituto Nacional de Cancerología.

Este proyecto contó con el aval del Comité de Investigación y Ética del Instituto Nacional de Cancerología y se respetaron los principios para la investigación en humanos enunciados en la Declaración de Helsinki, Informe Belmont y legislación colombiana.

\section{Resultados}

Entre agosto de 2013 y septiembre de 2015 se presentaron 665 pacientes en la unidad funcional de cáncer de seno del Instituto Nacional de Cancerología. De estas, 332 recibieron quimioterapia neoadyuvante y, de este último grupo, 72 pacientes tenían 65 años o más. El grupo final de 72 mujeres se describe en la tabla 1.

En cuanto a las variables demográficas, el promedio de edad de las evaluadas fue de 70,9 años. Más de la mitad de las pacientes tenían afiliación a EPS del régimen subsidiado.

Tabla 1. Características sociodemográficas y clínicas de la población incluida

\begin{tabular}{|c|c|}
\hline Característica & $\begin{array}{l}\text { Número de pacientes (\%) } \\
\qquad \mathbf{N}=\mathbf{7 2}\end{array}$ \\
\hline $\begin{array}{l}\text { EPS } \\
\text { Contributivo } \\
\text { Subsidiado }\end{array}$ & $\begin{array}{l}33(45,83) \\
39(54,17)\end{array}$ \\
\hline $\begin{array}{l}\text { ECOG inicial } \\
0 \\
1 \\
2\end{array}$ & $\begin{array}{c}18(25) \\
51(70,83) \\
3(4,17)\end{array}$ \\
\hline $\begin{array}{l}\text { ECOG final } \\
0 \\
1 \\
2 \\
3 \\
4 \\
4\end{array}$ & $\begin{array}{l}9(12,5) \\
55(76,39) \\
5(6,94) \\
2(2,78) \\
1(1,39)\end{array}$ \\
\hline $\begin{array}{l}\text { Charlson } \\
0 \\
1 \\
2 \\
3 \\
4\end{array}$ & $\begin{array}{c}46(63,89) \\
21(29,17) \\
3(4,17) \\
1(1,39) \\
1(1,39)\end{array}$ \\
\hline $\begin{array}{l}\text { Etapa clinica } \\
\| A \\
\text { IIB } \\
\text { IIA } \\
\text { IIB } \\
\text { IIIC }\end{array}$ & $\begin{array}{l}10(13,89) \\
10(13,89) \\
16(22,22) \\
32(44,44) \\
4(5,56)\end{array}$ \\
\hline $\begin{array}{l}\text { Tipo histológico } \\
\text { Ductal } \\
\text { Lobulillar } \\
\text { Otro }\end{array}$ & $\begin{array}{c}64(88,89) \\
3(4,17) \\
5(6,94)\end{array}$ \\
\hline $\begin{array}{l}\text { Grado histológico } \\
\text { Bien diferenciado } \\
\text { Moderadamente diferenciado } \\
\text { Pobremente diferenciado }\end{array}$ & $\begin{array}{c}3(4,17) \\
36(50,00) \\
33(45,83)\end{array}$ \\
\hline $\begin{array}{l}\text { Receptores hormonales } \\
\text { Positivos } \\
\text { Negativos }\end{array}$ & $\begin{array}{l}53(73,61) \\
19(26,39)\end{array}$ \\
\hline $\begin{array}{l}\text { HER2 } \\
\text { Positivo } \\
\text { Negativo }\end{array}$ & $\begin{array}{l}18(25,00) \\
54(75,00)\end{array}$ \\
\hline
\end{tabular}

Respecto a las variables de evaluación clínica encontramos que el $44,4 \%$ de las pacientes se encontraban en estadio IIIB al diagnóstico, seguido del IIIA con un $22,2 \%$. La histología predominante fue la ductal con un $88,8 \%$. En cuanto al estado de los marcadores se presentó positividad en los receptores hormonales $(\mathrm{RH})$ en un $73,6 \%$ y de HER2 en un $25 \%$.

Las pacientes se presentaron con un buen estado funcional. El 95,8\% de las pacientes que recibieron quimioterapia neoadyuvante tenían un ECOG al inicio de tratamiento de 1 o menos. Al finalizar dicho tratamiento este porcentaje disminuyó al $88,8 \%$. Solo 5 pacientes de 72 incluidas presentaron 2 o más puntos en la escala de Charlson.

Los esquemas de quimioterapia citotóxica utilizados y su frecuencia se muestran en la tabla 2.

De las 5 pacientes que solo recibieron AC como esquema neoadyuvante, 4 suspendieron su tratamiento

Tabla 2. Esquemas de quimioterapia neoadyuvante utilizados

\begin{tabular}{|l|c|}
\hline \multicolumn{1}{|c|}{ Esquema } & Frecuencia (\%) \\
\hline AC + paclitaxel & 45,8 \\
\hline AC + docetaxel & 11,1 \\
\hline AC exclusivo & 6,9 \\
\hline AC + paclitaxel carboplatino & 9,7 \\
\hline AC + docetaxel carboplatino & 1,3 \\
\hline Paclitaxel carboplatino & 8,3 \\
\hline Docetaxel carboplatino & 2,7 \\
\hline Docetaxel ciclofosfamida & 6,9 \\
\hline Paclitaxel solo & 4,1 \\
\hline Otros & 2,7 \\
\hline AC: doxorrubicina y ciclofosfamida &
\end{tabular}

por toxicidad grado 3 o 4. Ninguna de las pacientes que recibieron este protocolo alcanzó la respuesta patológica completa. La mediana de días entre la formulación del esquema y la aplicación de la primera dosis del protocolo fue de 13 días con un rango de 0 a 44 .

De las 72 pacientes incluidas, el $25 \%$ suspendió la quimioterapia neoadyuvante. Las causas evaluadas se muestran en la tabla 3.

Se usaron medicamentos de soporte en el 94,4\% de las pacientes. Dentro de estos fármacos los más

Tabla 3. Causas de suspensión del tratamiento neoadyuvante $(n=18)$

\begin{tabular}{|l|c|}
\hline \multicolumn{1}{|c|}{ Causa } & $\begin{array}{c}\text { Número } \\
\text { de pacientes (\%) }\end{array}$ \\
\hline Toxicidad grado 304 & $10(55,55)$ \\
Progresión de la enfermedad & $2(11,11)$ \\
Negativa del paciente & $4(22,22)$ \\
No autorización aseguradora & $1(5,55)$ \\
Otro & $1(555)$ \\
\hline
\end{tabular}


usados fueron los factores estimulantes de colonias en un $85,9 \%$ las pacientes, seguido de los inhibidores de neuroquinina 1 en el $75 \%$ de los casos. Se usó palonosetrón en una paciente.

Para el análisis de la intensidad de dosis, se excluyeron 2 pacientes a quienes se les formuló el tratamiento, pero no lo iniciaron por motivos no conocidos. En el grupo restante de 70 pacientes, el promedio de la intensidad de dosis de la quimioterapia neoadyuvante fue del $77 \%$, un $38,8 \%$ de las pacientes tuvieron una intensidad de dosis óptima (igual o superior al 85\%). Se realizó un análisis excluyendo las pacientes que suspendieron el tratamiento neoadyuvante, con lo cual el promedio de la intensidad de dosis aumentó a un $80,7 \%$, con un $40,7 \%$ de las pacientes alcanzando una intensidad de dosis óptima según se describió.

La respuesta clínica completa se evidenció en el $28,17 \%$ de las pacientes. En la evaluación por imagen, el $10,71 \%$ reportaron respuesta completa. El $84,7 \%$ de las pacientes incluidas fueron llevadas a cirugía de seno. De estas, el $32 \%$ fueron conservadoras y el $68 \%$ restante, cirugías radicales del seno. La tasa de respuesta patológica completa en nuestra cohorte fue de un $16,39 \%$ incluyendo todos los subtipos biológicos.

De las pacientes que tuvieron una intensidad de dosis óptima, un 17,8\% alcanzaron respuesta patológica completa versus un $11,3 \%$ de las que tuvieron una intensidad de dosis menor al 85\% $(p=0,49)$.

Un 75\% de las pacientes incluidas en nuestro estudio tenían tumores que expresaban receptores hormonales. La tasa de respuesta patológica completa en ellas fue del $11,36 \%$. En este grupo la intensidad de dosis promedio fue del $77,6 \%$, con un $46,1 \%$ de las mujeres alcanzando una intensidad de dosis óptima.

Todas las pacientes con tumores HER2 positivos recibieron trastuzumab, a excepción de una paciente que suspendió su tratamiento en la fase de antracíclicos. La intensidad de dosis de la terapia anti-HER2 en este grupo de pacientes fue del $77,9 \%$. El $23,5 \%$ de las pacientes en este grupo alcanzaron una respuesta patológica completa.

El $20,8 \%$ de las pacientes incluidas presentaron tumores triple negativos, el promedio de intensidad de dosis del grupo fue del $77,1 \%$. Casi todas las pacientes, a excepción de una, recibieron quimioterapia que incluía platinos. La tasa de respuesta patológica completa en este grupo fue de un $23 \%$.

\section{Discusión}

El cáncer de mama en el anciano presenta al clínico un contexto especial y diferente al de sus contrapartes más jóvenes. Este grupo reúne contradictoriamente la mayoría de las muertes y la mayoría de supervivientes de la enfermedad. Muchos pacientes con cáncer de mama se presentan con enfermedad localmente avanzada y es en este grupo en el que existe una mejor indicación para el tratamiento neoadyuvante ${ }^{23}$.

Con el presente estudio se perseguía efectuar un análisis descriptivo de pacientes mayores de 65 años que recibieron quimioterapia con intención neoadyuvante para cáncer de mama en el Instituto Nacional de Cancerología, con especial interés en la intensidad de dosis recibida y los aspectos que afectaban dicha variable. Nuestra cohorte reflejó el patrón de ocurrencia reportado en la literatura para el cáncer de mama en los ancianos con respecto al perfil demográfico y clínico típico ${ }^{6}$. No obstante, hubo hallazgos de interés que reflejan condiciones propias de nuestra población.

El promedio de edad registrado fue de 70 años. Se observó una baja carga de comorbilidades y un muy buen estado funcional al inicio del tratamiento, lo cual tal vez refleja el proceso de selección del paciente anciano que va a recibir quimioterapia como parte de su manejo. Es importante nombrar que menos del 7\% de los pacientes reportaron deterioro de su ECOG al finalizar la quimioterapia neoadyuvante, dato que es inferior a lo reportado por Shahrokni et al. en estudios de población geriátrica que recibe quimioterapia ${ }^{24}$.

De igual manera, encontramos que tan solo el $38,8 \%$ de las pacientes cumplieron con el estándar de intensidad de dosis del 85\% propuesto por Hryniuk, Banadonna y Valagussa ${ }^{19,20}$. Debido a que las pacientes que suspendían la quimioterapia de forma prematura representaban un sesgo en el cálculo de la fórmula (reciben menor dosis y a la vez finalizan su tratamiento en menos tiempo), se hizo un análisis adicional excluyéndolas, sin encontrar diferencias tanto en el promedio de la intensidad de dosis como en el porcentaje de pacientes con intensidad óptima.

Al evaluar la intensidad de dosis a la luz de otras variables demográficas o clínicas, encontramos una tendencia a presentar una intensidad de dosis no óptima en las pacientes de régimen subsidiado versus contributivo ( $59 \%$ vs. $40 \%$ ). Otros factores como el ECOG inicial, la escala de Charlson y el uso de 
medicamentos de soporte no mostraron diferencias respecto a alcanzar o no una intensidad de dosis óptima. Estos resultados podrían reflejar el bajo número de pacientes incluidas en nuestro estudio. No obstante, numéricamente estas últimas tres variables tampoco fueron diferentes, por lo cual intuimos que el resultado sería el mismo a pesar de tener un número mayor de participantes.

En cuanto a la respuesta clínica completa, este hallazgo se reportó en el $28,17 \%$ de los casos comparado con un $36 \%$ del NSABP $18^{25}$ y hasta un $63,6 \%$ con la adición de un taxano según el NSABP $27^{26}$. Es posible que esta cifra refleje un subreporte debido a las evaluaciones discrepantes entre las especialidades tratantes de este grupo, más aún, teniendo en cuenta que apenas un $10,71 \%$ de las pacientes evaluadas con imagen preoperatoria tenían un informe compatible con una respuesta completa por este método; dicha discordancia entre las respuestas patológica, clínica e imaginológica ya ha sido reportada previamente con resultados similares ${ }^{27}$.

La frecuencia de cirugías conservadoras de seno en nuestro grupo fue superior a la reportada en la literatura, hallazgo para tener en cuenta principalmente partiendo del hecho de que un $44,4 \%$ de pacientes se presentaron en estadio IIIB ${ }^{28}$.

Uno de los parámetros más importantes para la evaluación del impacto de la quimioterapia neoadyuvante es la frecuencia de respuesta patológica completa. En nuestro estudio usamos la definición de ausencia de tumor invasor en la mama y en los ganglios propuesta por Cortázar et al. en su metaanálisis de 201429. Nuestra frecuencia de respuesta patológica completa fue del $16,3 \%$, cifra inferior a la reportada por Cortázar del $18 \%$ incluyendo todos los subtipos biológicos.

En nuestra cohorte se encontró una tendencia a que las pacientes con intensidad de dosis inferior al $85 \%$ presentaran menor frecuencia de respuesta patológica completa versus aquellas con $85 \%$ o más ( $17,8 \%$ vs. $11,36 \%$ ).

Se realizó un análisis exploratorio combinando la intensidad de dosis y la respuesta patológica completa, según el perfil de marcadores (receptor hormonal positivo, HER2 positivo y triple negativo) y se halló que: las pacientes con tumores que expresaban receptores hormonales presentaron una respuesta patológica completa en el $11,3 \%$ de los casos, dato superior a lo reportado en la literatura $(7,6 \%)^{30}$. Nuestro reporte puede explicarse por la inclusión en este grupo de un $26,4 \%$ de pacientes con coexpresión de HER2 y receptor hormonal. De este $26,4 \%$, un $16 \%$ alcanzó respuesta patológica completa versus un $9,3 \%$ de las pacientes con expresión exclusiva de receptores hormonales. Si analizamos estos dos últimos datos y tenemos en cuenta que un $46,3 \%$ de estas pacientes tuvieron una intensidad de dosis óptima, podemos suponer que probablemente la intensidad de dosis es menos influyente en la respuesta patológica completa de los tumores con receptor hormonal positivo versus los que expresan también HER2.

Las pacientes con tumores que sobreexpresaban HER2 presentaron una respuesta patológica completa en el $23,5 \%$ de los casos, dato que es inferior al 30 $50 \%$ reportado en la literatura ${ }^{31,33}$. El porcentaje de pacientes con intensidad de dosis óptima fue del 38,8\% hallazgo que podría explicar la baja frecuencia de respuesta patológica completa observada en este grupo.

El grupo de pacientes con tumores triple negativos fue el que reportó el porcentaje más bajo de intensidad de dosis óptima con tan solo un 14\%. De la misma manera en este grupo, el porcentaje de pacientes que alcanzaron la respuesta patológica completa fue inferior a lo descrito en el metaanálisis de Cortázar et al. con apenas un $23 \%{ }^{29}$.

\section{Conclusiones}

Como fortalezas de nuestra investigación resaltamos 'en primer lugar' que el estudio está dirigido a una población que usualmente no se incluye en los estudios clínicos; y, en segundo lugar, el hecho de que evaluó un desenlace particularmente importante para esta población como es la respuesta patológica completa en relación con la intensidad de dosis, hallazgos previamente no explorados en el contexto de la quimioterapia neoadyuvante en los ancianos. Como desventajas encontramos el diseño retrospectivo, la escasa cantidad de pacientes, lo que pudo influir en no alcanzar las diferencias numéricamente importantes; y, por último, no tener una herramienta para eliminar el sesgo que produjeron las pacientes que abandonaron el tratamiento en el cálculo de la fórmula de la intensidad de dosis. En el análisis se eliminaron estas pacientes para dar mayor confiabilidad en los resultados.

Factores como el estado de receptores 
hormonales y HER2, el grado de diferenciación celular, el diámetro tumoral pretratamiento y la expresión de p53 se han descrito en los estudios como predictores de la respuesta al tratamiento neoadyuvante ${ }^{30,34}$. Como conclusión, nuestro estudio respalda las publicaciones previas que demuestran que los ancianos con frecuencia reciben un tratamiento subóptimo.

Si bien hay factores relacionados con la baja intensidad de dosis, es importante mencionar que variables como el grupo de edad, el estado funcional inicial, la tasa de comorbilidades o el uso de medicamentos de soporte no presentaron diferencias entre las pacientes que lograron una intensidad óptima versus las que no; se observó una tendencia a que los ancianos con intensidad de dosis baja mostraran menor respuesta patológica completa, en especial en los tumores más sensibles a la quimioterapia citotóxica.
Este hallazgo es de suma importancia, puesto que ya se ha demostrado la relación que existe entre la respuesta patológica completa y los desenlaces de supervivencia a largo plazo ${ }^{35}$.

La mejoría en la supervivencia de los enfermos con cáncer de mama y el cambio en la pirámide poblacional, nos obliga a conocer mejor las condiciones particulares del paciente anciano que padece de esta enfermedad. A pesar de ser un grupo de suma importancia por los factores antes descritos, se disponen de escasos estudios dirigidos a la población de 65 años o más que permitan hacer un manejo basado en la evidencia. El presente estudio pretende dar pie a investigaciones con un mayor número de pacientes y de diseño prospectivo analítico que ayude a generar hipótesis, para así reorientar el tratamiento de los ancianos con cáncer de mama hacia una práctica personalizada y adaptada a sus necesidades particulares.

\section{Referencias}

1. Bray F, Ferlay J, Soerjomataram I, et al. Global cancer statistics 2018: Globocan estimates of incidence and mortality worldwide for 36 cancers in 185 countries. CA Cancer J Clin. 2018 68:394-424.

2. Bray F, Ren J-S, Masuyer E, Ferlay J. Global estimates of cancer prevalence for 27 sites in the adult population in 2008. International Journal of Cancer. 2013;132(5):1133-45.

3. Colombia, Ministerio de Salud y Protección Social. Análisis de Situación de Salud (ASIS) Colombia 2015. Ministerio de Salud y Protección Social; 2015.

4. OMS. Informe mundial sobre el envejecimiento y la salud [Internet]. WHO. [cited 2016 jul 27]. Disponible en: http://www. who.int/ageing/publications/world-report-2015/es/

5. Smith BD, Smith GL, Hurria A, et al. Future of cancer incidence in the United States: burdens upon an aging, changing nation. J Clin Oncol. 2009;27:2758-65.

6. Karuturi M, VanderWalde N, Muss H. Approach and management of breast cancer in the elderly. Clin Geriatr Med. 2016;32(1):133-53.

7. VanderWalde A, Hurria A. Early breast cancer in the older woman. Clin Geriatr Med. 2012;28(1):73-91.

8. Schairer C, Mink PJ, Carroll L, Devesa SS. Probabilities of death from breast cancer and other causes among female breast cancer patients. J Natl Cancer Inst. 2004 sep 1;96(17):1311-21.

9. Goodwin JS. Factors affecting the diagnosis and treatment of older persons with cancer. In: Balducci L, Lyman GH, Ershler WB. Comprehensive geriatric oncology. London: Harwood; 1998. p. 115-21

10. Samet JM, Junt WC, Key C, Humble C, Goodwin JS. Choices of cancer therapy varies with age of patient. JAMA. 1986;255: 3385-90.

11. Turner NJ, Haward RA, Mulley GP, Selby PJ. Cancer in old age - is it inadequately investigated and treated? BMJ. 1999:319:309-12
12. Owusu C, Lash TL, Silliman RA. Effect of undertreatment on the disparity in age-related breast cancer-specific survival among older women. Breast Cancer Res Treat. 2007;102(2):227-36.

13. Allemani C, Storm H, Voogd AC, Holli K, Izarzugaza I, TorrellaRamos A, et al. Variation in "standard care" for breast cancer across Europe: a Eurocare-3 high resolution study. Eur J Cancer. 2010;46(9):1528-36.

14. Mauri D, Pavlidis N, loannidis JPA. Neoadjuvant versus adjuvant systemic treatment in breast cancer: a meta-analysis. J Natl Cancer Inst. 2005;97(3):188-94.

15. Van der Hage JA, Van de Velde CJ, Julien JP, Tubiana-Hulin $M$, Vandervelden C, Duchateau L. Preoperative chemotherapy in primary operable breast cancer: results from the European Organization for Research and Treatment of Cancer Trial 10902. J Clin Oncol. 200;19(22):4224-37.

16. Gralow JR, Burstein HJ, Wood W, Hortobagyi GN, Gianni L, von Minckwitz G, et al. Preoperative therapy in invasive breast cancer: pathologic assessment and systemic therapy issues in operable disease. J Clin Oncol. 2008;26(5):814-9.

17. Kaufmann M, Hortobagyi GN, Goldhirsch A, Scholl S, Makris $A$, Valagussa $P$, et al. Recommendations from an international expert panel on the use of neoadjuvant (primary) systemic treatment of operable breast cancer: an update. J Clin Oncol. 2006;24(12):1940-9.

18. Hryniuk W, Frei $E$, Wright FA. A single scale for comparing dose-intensity of all chemotherapy regimens in breast cancer: summation dose-intensity. J Clin Oncol. 1998;16(9):3137-47.

19. Bonadonna G, Valagussa P. Dose-response effect of adjuvant chemotherapy in breast cancer. N Engl J Med. 1981;304(1):10-5.

20. Hryniuk W, Levine MN. Analysis of dose intensity for adjuvant chemotherapy trials in stage II breast cancer. J Clin Oncol. 1986;4(8):1162-70 
21. Chirivella I, Bermejo B, Insa A, Pérez-Fidalgo A, Magro A, Rosello $S$, et al. Optimal delivery of anthracycline-based chemotherapy in the adjuvant setting improves outcome of breast cancer patients. Breast Cancer Res Treat. 2009;114(3):479-84.

22. Oladipo O, Coyle V, McAleer JJ, McKenna S. Achieving optimal dose intensity with adjuvant chemotherapy in elderly breast cancer patients: a 10-year retrospective study in a UK institution. Breast J. 2012;18(1):16-22.

23. Franceschini G, Terribile D, Magno S, Fabbri C, D'Alba PF, Chiesa $F$, et al. Update in the treatment of locally advanced breast cancer: a multidisciplinary approach. Eur Rev Med Pharmacol Sci. 2007;11(5):283-9.

24. Shahrokni A, Wu AJ, Carter J, Lichtman SM. Long-term toxicity of cancer treatment in older patients. Clin Geriatr Med. 2016;32(1):63-80.

25. Fisher B, Bryant J, Wolmark N, Mamounas E, Brown A, Fisher $E R$, et al. Effect of preoperative chemotherapy on the outcome of women with operable breast cancer. J Clin Oncol. 1998; 16(8):2672-85.

26. Bear HD, Anderson S, Brown A, Smith R, Mamounas EP, Fisher $B$, et al. The effect on tumor response of adding sequential preoperative docetaxel to preoperative doxorubicin and cyclophosphamide: preliminary results from National Surgical Adjuvant Breast and Bowel Project Protocol B-27. J Clin Oncol. 2003;21(22):4165-74.

27. Chagpar AB, Middleton LP, Sahin AA, Dempsey $P$, Buzdar AU, Mirza AN, et al. Accuracy of physical examination, ultrasonography, and mammography in predicting residual pathologic tumor size in patients treated with neoadjuvant chemotherapy. Ann Surg. 2006;243(2):257-64.

28. Semiglazov VF, Semiglazov VV, Dashyan GA, Ziltsova EK, Ivanov VG, Bozhok AA, et al. Phase 2 randomized trial of primary endocrine therapy versus chemotherapy in postmenopausal patients with estrogen receptor-positive breast cancer. Cancer. 2007;110(2):244-54
29. Cortazar $P$, Zhang L, Untch M, Mehta $K$, Costantino JP, Wolmark N, et al. Pathological complete response and long-term clinical benefit in breast cancer: the CTNeoBC pooled analysis. Lancet. 2014;384(9938):164-72.

30. Colleoni M, Viale G, Zahrieh D, Pruneri G, Gentilini O, Veronesi $P$, et al. Chemotherapy is more effective in patients with breast cancer not expressing steroid hormone receptors. Clin Cancer Res. 2004;10(19):6622-8.

31. Robidoux A, Tang G, Rastogi P, Geyer CE, Azar CA, Atkins JN, et al. Lapatinib as a component of neoadjuvant therapy for HER2-positive operable breast cancer (NSABP protocol B-41): an open-label, randomised phase 3 trial. Lancet Oncol. 2013 nov;14(12):1183-92

32. Gianni L, Eiermann W, Semiglazov V, Manikhas A, Lluch A, Tjulandin S, et al. Neoadjuvant chemotherapy with trastuzumab followed by adjuvant trastuzumab versus neoadjuvant chemotherapy alone, in patients with HER2-positive locally advanced breast cancer (the NOAH trial): a randomised controlled superiority trial with a parallel HER2-negative cohort. Lancet. 2010;375(9712):377-84.

33. Untch M, Loibl S, Bischoff J, Eidtmann H, Kaufmann M, Blohmer J U, et al. Lapatinib versus trastuzumab in combination with neoadjuvant anthracycline-taxane-based chemotherapy (GeparQuinto, GBG 44): a randomised phase 3 trial. Lancet Oncol. 2012;13(2):135-44.

34. Faneyte IF, Schrama J G, Peterse JL, Remijnse PL, Rodenhuis $S$, van de Vijver MJ. Breast cancer response to neoadjuvant chemotherapy: predictive markers and relation with outcome. $\mathrm{Br} J$ Cancer. 2003;88(3):406-12.

35. Broglio $K R$, Quintana $M$, Foster $M$, Olinger $M$, McGlothlin A, Berry SM, et al. Association of pathologic complete response to neoadjuvant therapy in HER2-positive breast cancer with long-term outcomes: a meta-analysis. JAMA Oncol. 2016;2(6):751-60. 